\section{Variabilidad metabólica asociada a índices de insulinorresistencia en pacientes de 20-35 años con sobrepeso atendidos en el Hospital General Los Esteros, Manta}

Metabolic variability associated with insulin-resistance indices in patients of 20-35 years with overweight care at the Los Esteros General Hospital, Manta.

Chamba Mero Bryan Alexander

Licenciado, Universidad Estatal del Sur de Manabí,

Portoviejo - ManaBí, Bchm21@hotmail.com

https://orcid.org/0000-0002-4257-3638

Zambrano Saltos Magaly Lisseth

Licenciada, Universidad Estatal del Sur de Manabí,

Portoviejo - Manabí, magalyzambrano-

96@outlook.es, https://orcid.org/0000-0002-

6343-6923

William Antonio Lino Villacreses

Magister, Universidad Estatal del Sur de Manabí,

Jipijapa - Manabi, william.lino@unesum.edu.ec,

https://orcid.org/0000-0001-5613-9958

\section{Guayaquil - Ecuador}

http://www.jah-journal.com/index.php/jah

Journal of American health

Enero - Marzo vol. 4. Num. 1 - 2021

Esta obra está bajo una Licencia Creative Commons

Atribución-NoComercial-CompartirIgual 4.0 Internacional.

RECIBIDO: 28 DE MAYO 2020

ACEPTADO: 12 DE NOVIEMBRE 2020

PUBLICADO: 4 DE ENERO 2021

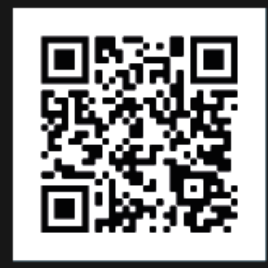

Scan this QR

code with your

smart phone or

mobile device to

read more papers

\section{RESUMEN}

El Síndrome Metabólico se lo conoce también como Variabilidad Metabólica denominado así por el conjunto de alteraciones metabólicas inmersas en distintos subgrupos de la obesidad, también se lo halla en la disminución de las concentraciones séricas del colesterol unido a altos potenciales de variantes de la Insulina, en el aumento de la presión arterial (PA) y en la hiperglucemia, todos para un diagnóstico necesario en el que los individuos presenten, al menos, tres de estas alteraciones metabólicas, características suficientes para obtener un alto índice de probabilidad en que aparezcan algunas de las enfermedades subyacentes. Objetivo: Determinar la variabilidad metabólica asociado a índices de Insulinorresistencia en pacientes de 2035 años con sobrepeso atendidos en el Hospital General Clínico Los Esteros del cantón Manta. Para el desarrollo del proyecto se utilizó investigación descriptiva correlacional de corte transversal empleando estudios analíticos, observacional y laboratorio. Metodología: Muestreo no probabilístico a conveniencia empleando los criterios de inclusión y exclusión a partir de encuesta, seleccionando a 123 pacientes que se les realizó las pruebas de Insulina y Glucosa, mediante método cinético y de ELISA. Resultados: Calculando la glucosa con 38 casos equivalentes a un 30,89\%, Índice de Cintura y Cadera con 7 casos equivalentes a 5,69\%; Presión arterial con un factor de 30 casos equivalentes a $24,39 \%$; Insulina con una alteración de 32 casos equivalentes $26,2 \%$. La correlación positiva respecto a la edad del índice HOMA-IR fue directamente proporcional al incremento de peso en edades comprendidas entre 28-31 años $(p=0,007)$, se observó $(p=0,006)$ con la 
presión, al nivel de insulina $(0,006)$, sin embargo no se observa correlación con el Índice de cintura y Cadera $(p=0,3)$. Conclusión: los trabajadores de reingreso laboral de los 123 casos presentaron alteraciones en: la glucosa; Presión arterial con un factor de $\mathbf{3 0}$ casos; Insulina con una alteración de 32 casos.

PALABRAS CLAVE: Variabilidad Metabólica, Homa-IR, Insulinorresistencia, Insulina, Elisa, Método Cinético, Presión Arterial.

\section{ABSTRACT}

The Metabolic Syndrome is also known as Metabolic Variability, named after the set of metabolic alterations immersed in different subgroups of obesity, it is also found in the decrease in serum cholesterol concentrations linked to high potentials of variants of Insulin, in the increase in blood pressure (BP) and in hyperglycemia, all for a necessary diagnosis in which individuals present at least three of these metabolic disorders, sufficient characteristics to obtain a high probability index in which some of the underlying diseases. Objective: To determine the metabolic variability associated with Insulin resistance rates in overweight patients aged $20-35$ years cared for at the General Clinical Hospital Los Esteros in the canton of Manta. For the development of the project, cross-sectional correlational descriptive research was used using analytical, observational and laboratory studies. Methodology: Convenient non-probability sampling using the inclusion and exclusion criteria from the survey, selecting 123 patients who underwent the Insulin and Glucose tests, using the kinetic and ELISA method. Results: Calculating glucose with 38 cases equivalent to $30.89 \%$, Waist and Hip Index with 7 cases equivalent to $5.69 \%$; Blood pressure with a factor of 30 cases equivalent to 24.39\%; Insulin with an alteration of 32 equivalent cases $26.2 \%$. The positive correlation with respect to age of the HOMA-IR index was directly proportional to the increase in weight at ages between 28-31 years $(p=0.007)$, it was observed ( $p=0.006$ ) with pressure, at the insulin level (0.006), however, no correlation was observed with the Waist and Hip Index $(p=0.3)$. Conclusion: the reentry workers of the 123 cases presented alterations in: glucose; Blood pressure with a factor of 30 cases; Insulin with an alteration of 32 cases.

KEYWORDS: Metabolic Variability, HomaIR, Insulin resistance, Insulin, Elisa, Kinetic Method, Blood Pressure.

\section{INTRODUCCIÓN}

El Síndrome Metabólico se lo conoce también como variabilidad metabólica, denominado así por el conjunto de alteraciones metabólicas inmersas en distintos subgrupos de la obesidad, también se lo halla en la disminución de las concentraciones séricas del colesterol unido a las lipoproteínas de alta densidad, en el aumento de las concentraciones de triglicéridos, en el aumento de la presión arterial (PA) y en la hiperglucemia, todos para un diagnóstico necesario en el que los individuos presenten, al menos, tres de estas alteraciones metabólicas, 
características suficientes para obtener un alto índice de probabilidad en que aparezcan algunas de las enfermedades subyacentes. (1)

El predominio de variabilidad metabólica se detalla como un conjunto de factores de riesgo, que requieren la presencia de obesidad como problema central para el asocio de otras enfermedades como: dislipemias, hipertensión arterial y resistencia a la insulina, todos ellos, predictores de patologías cardiovasculares y diabetes tipo 2 en un futuro, previos a estudios correlaciónales se demuestran que el índice de síndrome metabólico es dependiente de las definiciones utilizadas, observando una prevalencia mayor en la región mediterránea que en la zona Sur y norte de América. (1)

Factores de riesgos del síndrome metabólico

Hipertensión Arterial

Uno de los factores predominantes para la detección de variabilidad metabólica es la Hipertensión Arterial (HTA) que continúa siendo un problema de Salud Pública relevante a nivel mundial, este, se caracteriza por ser una de las principales causas de morbimortalidad y un factor de riesgo de mayor carga atribuible para la patología cardiovascular isquémica y el accidente cerebrovascular, se resaltan sus prevalencias, el grado de conocimiento, el logro de su control y tratamiento, así como su riesgo atribuible analizando la carga de enfermedad y la pérdida de años por discapacidad. Se presentan estudios del impacto de su inclusión en el AUGE, resultados de la intervención multidisciplinaria, su interacción con otros factores de riesgo cardiovascular y se comentan algunos aspectos de las actualizaciones de las guías internacionales para su manejo. (2)

Hiperglicemia.

Uno de los factores medibles por analitos en la resistencia a la insulina se produce cuando las células de los músculos, grasa e hígado no responden bien a la insulina y no pueden absorber la glucosa de la sangre fácilmente ocasionando entre uno de los problemas detectables las dislipidemias que son cada vez más frecuentes en el ser humano, al igual que la hiperglicemia, debido al inadecuado estado nutricional de las personas, caracterizada por tener el azúcar en la sangre, también llamada glucosa es el azúcar inicial que se encuentra en la sangre, su ubicación principal es en el organismo ya que es uno de los alimentos de ingesta diaria y es una de las principales fuentes de energía del cuerpo y a pesar de que los niveles de glucosa en la sangre no son lo suficientemente altos como para causar síntomas en la mayoría de las personas, algunos estudios de investigación han mostrado que algunas personas con prediabetes podrían ya tener cambios tempranos en los ojos que pueden conducir a la retinopatía. Este problema ocurre con más frecuencia en personas con diabetes. (3)

Exceso de Cintura y Cadera

Tomando como características primordiales de los autores nos informan que el índice cintura/cadera se presenta como un elemento más de la valoración clínica de la obesidad y sus consecuencias que se buscó describir la utilidad del empleo del índice cintura/cadera en la detección del riesgo cardiometabólico durante su proceso utilizaron métodos como el Google Académico como motor de búsqueda y se obtuvo un total de 97 artículos, de los cuales 61 fueron referenciados por cumplir con el objetivo enunciado y obtuvieron como resultados que la obesidad se comporta como una enfermedad multifactorial, de evolución crónica, con importantes consecuencias para la salud. El incremento del tejido adiposo abdominal, propicia una mayor síntesis y liberación de adipoquinas y otras sustancias, que pueden deteriorar el 
metabolismo lipídico y glucídico a través del aumento de la resistencia a la insulina, e incrementan el riesgo cardiovascular. (4).

\section{MATERIALES Y MÉTODOS}

Método deductivo-inductivo: permitió conocer y analizar la problemática. Método descriptivo y bibliográfico: donde se realiza la descripción bibliográfica de las conceptualizaciones teóricas. Método estadístico: permitió analizar, interpretar, presentar los resultados obtenidos de las encuestas, Investigación no experimental: basándose en la observación para posteriormente ser analizada y conceptualizada. Investigación bibliográfica: requeridas durante la investigación tales como: libros y páginas virtuales de investigaciones médicas, investigación de observación de campo: con la finalidad de conocer la realidad de la problemática del entorno en el proyecto. Técnicas e instrumentos, la técnica utilizada en la investigación fue la encuesta a los pacientes que acuden al consultorio externo del servicio de laboratorio del hospital general clínicas los Esteros del Cantón Manta, con la finalidad de obtener información relacionada a la problemática. Los instrumentos utilizados son: formato de las encuestas.

Análisis de muestras: mediante Elisa y Espectrofotometría.

Encuesta: Este instrumento se aplicó a una muestra de 123 pacientes que acuden al Hospital Clínica los Estero, para recopilar información necesaria para la elaboración de la investigación. Así mismo se aplicó a los 123 pacientes exámenes de laboratorio y tomas de muestras que nos permitan conocer los resultados clínicos de niveles de glucosa e insulina.

Técnicas de laboratorio, llevando a cabo la fase pre-analítica, analítica y post- analítica se lograra obtener datos coinfiables. La fase analítica para el procesamiento de las muestras se realizara a través de los métodos espectrofotometría Se realizará el cálculo de Índice De Masa Corporal (IMC) e ICC.

\section{RESULTADOS}

En la población de muestreo hubo una prevalencia de las siguientes características clínicas; Antecedentes familiares con 5 patologías suscritas con: Diabetes con 37 casos $(30,1 \%)$, Hipertensión 44 casos (35,8\%), Cáncer 7 casos (5,7\%), Hepatitis 2 casos (12.0\%); Constan de un cargo Actual donde: 37 (30,1\%) personas corresponden al área de comedor y $86(69,9 \%)$ personas al área de operario; Clasificación Nutricional con: Eutróficos $3(2,4 \%)$ casos, obesos 3 $(2,4 \%)$ casos y sobrepeso con $117(95,1 \%)$ casos, Estado de la glucosa con: Glicemia normal 82 $(66,7 \%)$ casos e Hiperglicemia 41 (33,3\%) casos; Factor Presión con: Hipotensión 5 (4,1\%) casos, Presión Normal: 60 (48,8\%) casos e Hipertensión 58 (47,2\%) casos.

Los valores de los pacientes indican que la variabilidad metabólica asociada a índices de insulinorresistencia dieron como resultado en alteraciones inmersas en: La glucosa con 38 casos equivalentes a un $30,89 \%$, Indice de Cintura y Cadera con 7 casos equivalentes a 5,69\%; Presión arterial con un factor de 30 casos equivalentes a 24,39\%; Insulina con una alteración de 32 casos equivalentes $26,2 \%$; Todos estas alteraciones demostradas con 117 pacientes con sobrepeso equivalente $95,09 \%$ y 3 casos para obesidad y 3 casos para eutróficos equivalentes al 2,4\% del total de población.

Se pudo conocer mediante el examen del Indice de Homar-ir que esta prueba es primordial en el Hospital General Clínico Los esteros ya que mediante la clasificación referente al sexo: femenino con un promedio de 69 personas, 17 casos equivalentes a 13,82\% nos dieron con 
resistencia a la insulina y con sexo Masculino con un promedio de 54 personas, 21 casos equivalentes al $17,07 \%$ nos dieron con resistencia a la insulina acoplándose con mayor prevalencia de casos de resistencia a la insulina en el lugar de muestreo.

De las 38 personas con Variabilidad Metabólica la edad que predomino de acuerdo a la resistencia a la insulina fue de 28-31 años, en donde 18 personas presentaron resistencia a la Insulina que corresponde al 47,4\%, y 23 casos presentaron variabilidad metabólica sin resistencia a la insulina que corresponde $27,1 \%$.

\section{DISCUSIÓN}

La correlación positiva respecto a la edad del índice HOMA-IR fue directamente proporcional al incremento de peso de los estudiantes mediante la significancia medida por el chip de Pearson les dio como resultado $(p=0,01)$, se observó $(p=0,018)$, al nivel de insulina $(0,001)$, sin embargo no se observa correlación con el nivel de glicemia $(p=0,5)$ a diferencia de nuestros resultados que en pacientes de 28 a 31 años, de los cuales 18 personas desarrollaron alteraciones en la glucosa con ( $p=0,000007)(5)$

La relación entre índices de resistencia a la insulina e hipertensión en los pacientes brindó una significancia de $(p=0,0008)$ brindando un porcentaje de $24,39 \%$ de los casos en estudio demostrando que hay asociación entre ambos factores.

Los autores redactan que el estudio en pacientes no diabéticos se realizó la prueba del índice de HOMA-IR en que les dio como resultado en el e ISI-Composite ( $r=-089, p<0.001)$, se propone, que los niveles de insulina sérica de 60 y 120 minutos sean de 130 y $80 \mu \mathrm{U} / \mathrm{ml}$ respectivamente, como valores de corte para la normalidad en individuos normoglucémicos mientras que en el estudio que se brindó en la investigación el índice de Homa- ir se lo clasifico mediante el sexo donde se obtuvo como resultado que el género femenino abarca un porcentaje de $13,82 \%$ mientras que en el género masculino brindo un porcentaje de $17,07 \%$ en pacientes hiperglicemicos.

En investigaciones previas descritas por los autores en pacientes con SM $(n=89)$ y sin SM $(n=436)$, en donde las capacidades discriminativas para la evaluación del SM y del índice de masa corporal, calculado como $\mathrm{Ln}(\mathrm{TG}[\mathrm{mg} / \mathrm{dL}]$ x glucosa[mg/dL]/2), y de la relación TG/C-HDL y la probabilidad pretest para $\mathrm{SM}=30 \%$ con una equidad de estudios descritos en base a los criterios de sobrepeso con un porcentaje del 30,89\% de mayor prevalencia en distinción a la obesidad, eutróficos.

\section{CONCLUSIONES}

Una vez culminado con el estudio sobre la correlación entre los niveles de glucosa e insulina y el índice de masa corporal e icc en personas de 20 a 35 años de edad de pacientes que asisten al hospital general clínico los esteros se establecen las siguientes conclusiones:

Para el cumplimiento del primer objetivo se ha determinado que la Variabilidad Metabólica asociada a índices de Insulinorresistencia en los trabajadores de reingreso laboral con un total de los 123 casos presentaron alteraciones en la glucosa, presión arterial y en el examen de sensibilidad a la insulina; alteraciones demostradas en pacientes con criterios de sobrepeso, obesidad y eutróficos respectivamente. 
De acuerdo a las características clínicas predominaron 5 patologías por Antecedentes Familiares suscritas; Diabetes, Hipertensión, Cáncer, Hepatitis, todos ellos pertenecientes a una actividad laboral correspondientes al área de comedor y áreas operativas.

El examen del Índice de Homar-ir prueba que es primordial implementarla en laboratorios clínicos por la importancia que tiene para la identificación de resistencia a la insulina con relación al sexo, el cual ha demostrado que no existe distinción entre masculino y femenino más sin embargo afecta a ambos sexos sin distinción.

Según la Resistencia a la insulina y edad se demostró que las personas a quienes se les pudo determinar Variabilidad Metabólica y cuyas condiciones presentaban como factor de riesgo el sobrepeso se presentó en edades de 28-31 años.

Según el aporte que brinda esta investigación se concluyó mediante el enfoque contributario e informativo a la comunidad en general, generando una información relevante para el campo científico y con una nueva línea de datos actualizados sobre estas enfermedades.

\section{REFERENCIAS}

1. Paul Z. Una nueva definicion mundial del sindrome metabolico. Revista Esppañola de Cardiologia. 2015; 58(12).

2. Miriam P. Factores indirectos de la obesidad. Revista Medica Española. 2016; 44(23).

3. Miguel MR. Prevalencia Mundiaol de Diabetes Mellitus tipo 2 y su relacion con el indice de desarrollo humano. Revista Panamericana de la Salud Publica. 2018; 56(3).

4. Freire WB, Ramírez-Luzuriaga MJ, Belmont $\mathrm{P}$, Mendieta MJ, Silva-Jaramillo K, Romero N. Encuesta Nacional de Salud y Nutricion. [Online]; 2014.

5. Maria C. Prevalencia de obesidad en Ecuador. Manual del Modelo de Atencion Intergral de Salud MAIS. 2016; 210(5).

6. Luis P. Prevalencia de Diabetes Mellitus. Revista Panamericana de la Salud. 2017; 15(3).

7. Manuel PR. Obeisdad, adipogenesis y resistencia a la insulina. Revista Medica Las Condes. 2016; 58(7).

8. Calvo MC. EL SÍNDROME METABÓLICO. Universidad Complutense. 2018.

9. Fernández VG. Base de datos aplicada a la atención farmacéutica en la oficinas de farmacia Tesis Doctoral. Madrid: UNIVERSIDAD COMPLUTENSE DE MADRID, Departamento de Farmacia y Tecnología Farmacéutica.

10. Organizacion Mundial de La Salud. Informe mudial sobre la diabetes. [Online]; 2016. 
11. Mora RIÁ. CUIDADOS DE ENFERMERÍA EN PACIENTES CON PIE DIABÉTICO EN CENTRO DE SALUD MANTA. Tesis Licenciatura. Manta: Universidad Laica Eloy Alfaro de Manabí., Repositorio Uleam.

12. Nexar Vicente Laz Vera RTD. ACTIVIDADES FÍSICAS RECREATIVAS Y SU INCIDENCIA EN LOS NIVELES DE OBESIDAD. CASO DE ESTUDIO UNIDAD EDUCATIVA RÉPLICA MANTA. Revista Electronica formacion y calidad educativa. 2018; 6(3).

13. Citlaly Gutiérrez-Rodelo ARGyJAOR. Mecanismos Moleculares de la Resistencia a la Insulina. Pubmed. 2017.

14. Angosto MSRyMC. RESISTENCIA A LA INSULINA, INFLAMACION Y OBESIDAD . Scielo. 2015.

15. Benalcázar Cachimuel EM. Factores de riesgo en síndrome metabólico relacionado con hábitos alimentarios y actividad física en los adultos del barrio Tanguarín, San Antonio de Ibarra, 2017. Universidad tecnica del norte. 2018.

16. José Hernández Rodríguez OMMEYA. Utilidad del índice cintura/cadera en la detección del riesgo cardiometabólico en individuos sobrepesos. Revista Cubana de Endocrinología. 2018.

17. Teherán AA. Poliuria. researchgate. 2018.

18. Karen J. Adams JLC. prevalencia de factores de riesgo para sindrome metabolico y sus componentes en usuarios de comedores de Lima, Peru. scielo. 2018. 